\title{
Multi element synthetic aperture transmission using a frequency division approach
}

\author{
Gran, Fredrik; Jensen, Jørgen Arendt
}

Published in:

IEEE Symposium on Ultrasonics, 2003

Link to article, DOI:

10.1109/ULTSYM.2003.1293297

Publication date:

2003

Document Version

Publisher's PDF, also known as Version of record

Link back to DTU Orbit

Citation (APA):

Gran, F., \& Jensen, J. A. (2003). Multi element synthetic aperture transmission using a frequency division approach. In IEEE Symposium on Ultrasonics, 2003 (Vol. 2, pp. 1942-1946). IEEE.

https://doi.org/10.1109/ULTSYM.2003.1293297

\section{General rights}

Copyright and moral rights for the publications made accessible in the public portal are retained by the authors and/or other copyright owners and it is a condition of accessing publications that users recognise and abide by the legal requirements associated with these rights.

- Users may download and print one copy of any publication from the public portal for the purpose of private study or research.

- You may not further distribute the material or use it for any profit-making activity or commercial gain

- You may freely distribute the URL identifying the publication in the public portal 


\title{
Multi Element Synthetic Aperture Transmission using a Frequency Division Approach
}

\author{
Fredrik Gran and Jørgen Arendt Jensen \\ Center for Fast Ultrasound Imaging, Ørsted•DTU, Bldg. 348, \\ Technical University of Denmark, DK-2800 Kgs. Lyngby, Denmark
}

\begin{abstract}
In synthetic aperture imaging an image is created by a number of single element defocused emissions. A low resolution image is created after every emission and a high resolution image is formed when the entire aperture has been covered. Since only one element is used at a time the energy transmitted into the tissue is low.

This paper describes a novel method in which the available spectrum is divided into $2 N$ overlapping sub-bands. This will assure a smooth broadband high resolution spectrum when combined. The signals are organized into two subsets in which all signals are fully orthogonal. The transmitting elements are excited so that $N$ virtual sources are formed. All sources are excited using one subset at a time. The signals can be separated by matched filtration, and the corresponding information is extracted. The individual source information is hence available in every emission and the method can therefore be used for flow imaging, unlike space time encoding techniques such as Hadamard and Golay encoding. The frequency division approach increases the SNR by a factor of $N^{2}$ compared to conventional pulsed synthetic aperture imaging, provided that $N$ transmission sources are used. Simulations and phantom measurements are presented to verify the method.
\end{abstract}

\section{INTRODUCTION}

In conventional synthetic aperture imaging (STA) [1] an image is created by making a number of consecutive defocused transmissions from different locations of the aperture. The purpose is to form one image for every single element transmission. This is accomplished by beamforming the RF-lines originating from each transmission. However, the individual images have low resolution, since the corresponding transmission only covers a small part of the aperture. By adding the low resolution images, an image with higher resolution can be constructed. The main advantage of STA is that perfect focus can be attained for all points in the image, since RFdata from the individual defocused transmissions are available. The image has to be acquired over a number of emissions, and motion can therefore be a problem. Since only a small part of the aperture is used in every transmission, conventional STA provides a lower SNR than linear array imaging. The SNR can be increased by using several elements in transmit to form the spherical wave [2]. This can also be combined with a chirp excitation [3] to increase the SNR beyond that of a conventional image. The basic problem however, is that only part of the aperture can be used during transmission. This can be solved by using space time encoding [4] in which the full aperture can be used. These techniques, relies on the tissue to be stationary and can therefore not be used for flow imaging, where the SNR is most crucial.

In this paper we propose a novel method in which all transmitting sources can be excited at the same time and still be separated at the receiver, see Fig. 1. This is done by defining a set of narrow-band signals, which are disjoint in the frequency domain, enabling the receiver to separate the different signals. Since the individual signals are narrow-band, it is imperative to do a number of consecutive firings, so that each source transmits every signal in the set and thereby cover the entire bandwidth of the transducer. To synthesize a smooth broadband spectrum, it is necessary to define two sets of signals, which are overlapping but with signals mutually orthogonal within the sets, as will be shown in Section II. If the system consist of $N$ sources, $2 N$ signals have to be defined and $2 N$ transmissions have to be made. The approach will improve the SNR, which will be shown in Section III, and data from all sources are available in every transmission unlike space time encoding making it suitable for flow imaging. In Section IV some simulation and experimental results are shown and concluding remarks are given in Section V.

\section{MethoD}

The transmitting aperture is excited so that $N$ virtual sources are formed. Each source must be assigned an unique signal in order to separate all signals at the receiver. This means that in every transmission there has to be at least $N$ signals. Let

$$
\left\{s_{k}(t)\right\}_{k=1}^{N} \leftrightarrow\left\{S_{k}(f)\right\}_{k=1}^{N}
$$



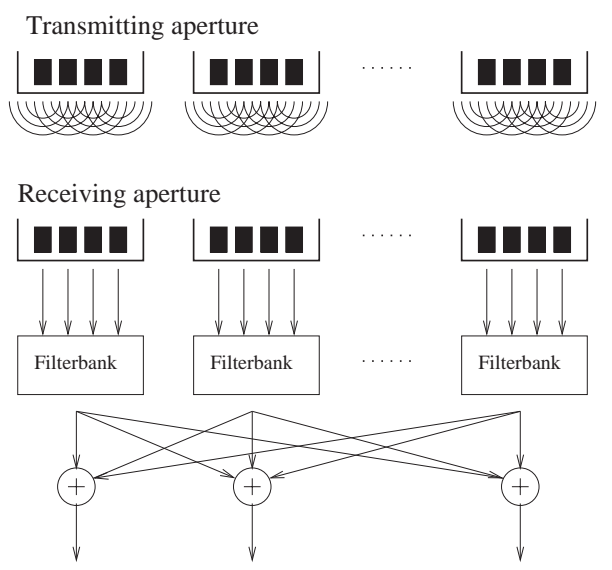

Beamforming low resolution images

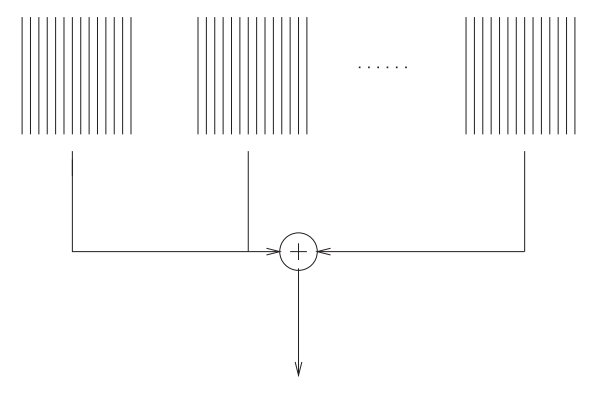

High resolution image

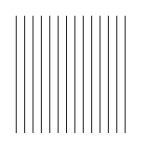

Figure 1: Frequency division synthetic aperture imaging

denote the signals, which are transmitted and their corresponding Fourier transforms. Under the assumption that the system is governed by the linear wave-equation, the response at the $j$ :th receiver can be modeled as,

$$
R_{j}^{(n)}(f)=\sum_{i=1}^{N} H_{i j}(f) S_{k(i, n)}(f)
$$

where $H_{i j}(f)$ corresponds to the pulse echo frequency response between the $i$ :th transmitting source and the $j$ :th receiving element and $S_{k(i, n)}(f)$ is the signal transmitted on the $i$ :th source at the $n$ :th transmitting event. To be separable by a matched filter at the receiver, the signals have to have the following property,

$$
S_{k}(f) S_{l}^{*}(f)=0, \forall k \neq l,
$$

where $S_{l}^{*}(f)$ is the complex conjugate of $S_{l}(f)$. This condition will automatically be fulfilled, if the signals do not overlap in the frequency domain, see Fig. 2. The signals will thus have to be band limited. Consider a frequency response function $S(f)$ which vanishes outside of $f_{0}+\frac{f_{B}}{2}>f>f_{0}-\frac{f_{B}}{2}$ where $f_{0}$ is the center frequency and $f_{B}$ is the total bandwidth

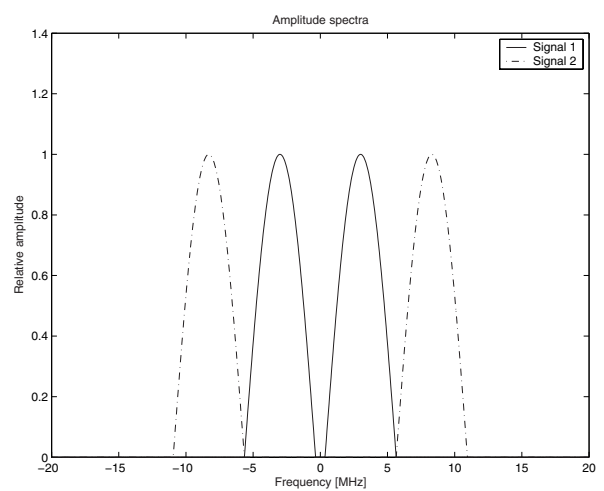

Figure 2: Two signal spectra, disjoint in the frequency domain

of the corresponding signal. A set of signals disjoint in the frequency domain can now be defined as

$$
S_{k}(f)=S\left(f-(k-1) f_{B}\right), k=1 \ldots N .
$$

If the $i$ :th source transmits signal with index $k(i, n)$ the filtered contribution at the $j$ :th receiver will be,

$$
R_{i j}^{(n)}(f)=R(f)_{j}^{(n)} S_{k(i, n)}^{*}(f)=H_{i j}(f)\left|S_{k(i, n)}(f)\right|^{2} .
$$

Since the result from (5) is narrow-band, the system would yield a very poor resolution if only one transmission was made. Therefore it is necessary to transmit with all signals on all sources to synthesize a smooth broadband spectrum. The resulting received spectrum from the $i$ :th source will then be given by,

$$
R_{i j}^{t o t}(f)=\sum_{n=1}^{N} R_{i j}^{(n)}(f)=H_{i j}(f) \sum_{n=1}^{N}\left|S_{k(i, n)}(f)\right|^{2} .
$$

The variable $k(i, n)$ is both a function of transmitting source number $i$, and transmit event $n$. It is desirable that $\sum_{n=1}^{N}\left|S_{k(i, n)}(f)\right|^{2}$ is a smooth function under the bandwidth of the frequency response function $H_{i j}(f)$, so that the achieved image quality is as good as possible. However it is unrealistic to assume that $\left\{S_{k}(f)\right\}_{k=1}^{N}$ can be defined so that $\left\{\left|S_{k}(f)\right|^{2}\right\}_{k=1}^{N}$ are rectangular functions, instead the signal spectra have to be continuous as in Fig. 2. This makes it necessary to define a second set of signals overlapping the set given in (1). Let

$$
\left\{s_{k}(t)\right\}_{k=N+1}^{2 N} \leftrightarrow\left\{S_{k}(f)\right\}_{k=N+1}^{2 N}
$$

represent the second set of signals and their corresponding Fourier transforms. If the new set of signals is chosen such that

$$
\left\{S_{k}(f)\right\}_{k=N+1}^{2 N}=\left\{S_{k}\left(f-\frac{f_{B}}{2}\right)\right\}_{k=1}^{N}
$$




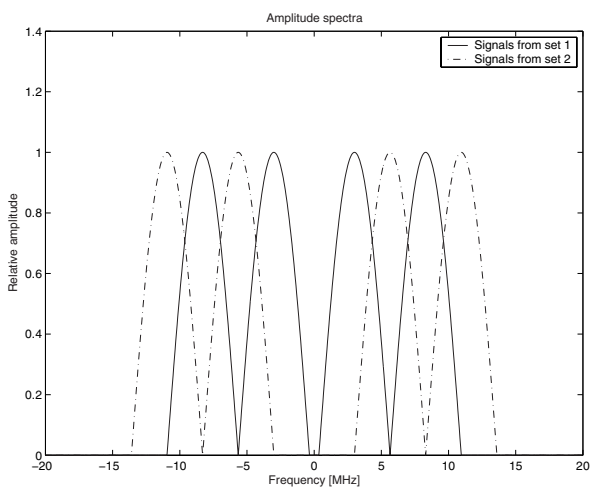

Figure 3: Two overlapping sets of spectra

the two different sets will overlap exactly. An example with 2 sources and 4 signals can be seen in Fig. 3. One can choose the functions $\left\{S_{k}(f)\right\}_{k=1}^{2 N}$, so that $\sum_{k=1}^{2 N}\left|S_{k}(f)\right|^{2}$ is a constant function on the interval $f_{0} \leq f \leq f 0+f_{B} \frac{2 N-1}{2}$. The total received signal for the $2 N$ transmissions at the $j$ :th receiver originating from the $i$ :th source can now be written as

$$
R_{i j}^{t o t}=H_{i j}(f) \sum_{n=1}^{2 N}\left|S_{k(i, n)}(f)\right|^{2}
$$

Since $\sum_{k=1}^{2 N}\left|S_{k}(f)\right|^{2}$ is a smooth broadband function, the result from (9) will also be broadband and a good image quality can be attained. It is finally worth repeating that for a system consisting of $N$ sources there will be $2 N$ signals and thus $2 N$ transmissions are necessary, since signals from the set given in (1) must not be transmitted at the same time as signals from the set given in (7). A typical firing scheme for a system with 4 sources is given in Table 1 .

\begin{tabular}{c|cccccccc}
$k(i, n)$ & \multicolumn{10}{|c}{$n$} \\
$i$ & 1 & 2 & 3 & 4 & 5 & 6 & 7 & 8 \\
\hline 1 & 1 & 2 & 3 & 4 & 5 & 6 & 7 & 8 \\
2 & 4 & 1 & 2 & 3 & 8 & 5 & 6 & 7 \\
3 & 3 & 4 & 1 & 2 & 7 & 8 & 5 & 6 \\
4 & 2 & 3 & 4 & 1 & 6 & 7 & 8 & 5
\end{tabular}

Table 1: Firing sequence for the total signal set

\section{SIGNAL-TO-NOISE RATIO}

In this section the effects of the frequency division multi source communication technique will be discussed. Consider a setup where the received signals originates from one point scatterer. The noise is assumed to be white additive and to have a gaussian distribution. First a system which operates according to a conventional STA firing scheme will be analyzed. The system consists of $N$ sources and for fair comparison $2 N$ transmissions are made. Consider the data from one single source transmission yielding one low resolution image. The power of the noise in this low resolution image is then,

$$
P_{l o}^{\text {conv }}=\int_{-\infty}^{\infty} P_{0}|H(f)|^{2} d f=P_{0} E_{h}
$$

where $P_{0}$ is the power spectral density of the noise, $H(f)$ is the frequency response and $E_{h}$ is the energy of the transmitted waveform. The total power of the noise for the $2 N$ low resolution images is,

$$
P_{h i}^{c o n v}=2 N P_{0} E_{h},
$$

assuming that the noise is uncorrelated from emission to emission. The peak output of the filtered transmitted signal in one low resolution image is [5]

$$
y_{\text {lo }}^{\text {conv }}\left(t_{\text {max }}\right)=\int_{-\infty}^{\infty}|H(f)|^{2} d f=E_{h} .
$$

The high resolution image will then give the peak output

$$
y_{h i}^{c o n v}\left(t_{\max }\right)=2 N E_{h},
$$

when all low resolution images are combined coherently. The peak pulse signal to noise ratio is then

$$
S N R_{\text {conv }}=\frac{\left|y_{h i}^{\text {conv }}\left(t_{\text {max }}\right)\right|^{2}}{P_{h i}^{\text {conv }}}=2 N \frac{E_{h}}{P_{0}} .
$$

If the energy of each signal is denoted $E_{s}$ for the frequency division case, the noise power of one low resolution image is given by

$$
P_{l o}^{F D}=\sum_{k=1}^{2 N} \int_{-\infty}^{\infty} P_{0}\left|S_{k}(f)\right|^{2} d f=2 N P_{0} E_{s},
$$

which gives that the noise power of the high resolution image is

$$
P_{h i}^{F D}=2 N^{2} P_{0} E_{s}
$$

The total peak output of a low resolution image is

$$
y_{l o}^{F D}\left(t_{\max }\right)=\sum_{k=1}^{2 N} \int_{-\infty}^{\infty}\left|S_{k}(f)\right|^{2} d f=2 N E_{s},
$$

and the peak output of the corresponding high resolution image is

$$
y_{h i}^{F D}\left(t_{\max }\right)=2 N^{2} E_{s} .
$$

This gives

$$
S N R_{F D}=\frac{\left|y_{h i}^{F D}\left(t_{\max }\right)\right|^{2}}{P_{h i}^{F D}}=2 N^{2} \frac{E_{S}}{P_{0}} .
$$


This again assumes that the noise is uncorrelated from emission to emission and that the summing of the low resolution images is coherent. Therefore it can be stated that

$$
\frac{S N R_{F D}}{S N R_{\text {conv }}}=N \frac{E_{S}}{E_{h}} .
$$

If a normal pulsed STA system is analyzed as a reference, $E_{h}=\frac{a^{2}}{2} T_{h}$ and $E_{s}=\frac{a^{2}}{2} T_{s}$ where $a$ is the amplitude and $T_{h}$ and $T_{s}$ are the duration of the corresponding waveforms. The duration of the waveforms are related to the bandwidths $B_{s}$ and $B_{h}$ as $T_{s} \sim \frac{1}{B_{s}}$ and $T_{h} \sim \frac{1}{B_{h}}$. The bandwidths are related as $B_{s} \sim \frac{B_{h}}{N}$ which gives,

$$
\frac{E_{s}}{E_{h}}=N
$$

so that

$$
\frac{S N R_{F D}}{S N R_{\text {conv }}}=N^{2} .
$$

When a chirp excitation is used however, the duration of the chirp can be chosen to equal the durations of the frequency division signals. This reduces the gain in SNR to

$$
\frac{S N R_{F D}}{S N R_{\text {conv-chirp }}}=N .
$$

using 8 transmission sources will, thus increase the signal-tonoise ratio by $9 \mathrm{~dB}$.

\section{Simulations AND MEASUREMENTS}

The method was tested using both simulations in Field II [6] and experiments on the experimental ultrasound scanner RASMUS [7]. Field II was used to simulate an ultrasound system with 64 transmitting and 128 receiving elements. The transmit aperture was organized so that 8 virtual sources were formed. The target was a single scatterer located $40 \mathrm{~mm}$ from the transducer surface. The transducer center frequency was $7 \mathrm{MHz}$ with a kerf of $0.035 \mathrm{~mm}$ and pitch of $0.208 \mathrm{~mm}$. First the conventional STA technique with a 1 cycle, $7 \mathrm{MHz}$ sinusoid was used to simulate the point spread function (PSF) as a reference. The result can be seen in Fig. 4. The axial resolution was $0.21 \mathrm{~mm}(-3 \mathrm{~dB})$ and $0.31 \mathrm{~mm}(-6 \mathrm{~dB})$ respectively, and the lateral resolution was $0.41 \mathrm{~mm}(-3 \mathrm{~dB})$ and $0.62 \mathrm{~mm}$ $(-6 \mathrm{~dB})$. The same setup was used for the frequency division approach with 16 bands starting from $1.5 \mathrm{MHz}$ to $17.5 \mathrm{MHz}$. The PSF is given in Fig. 5. The axial resolution was $0.23 \mathrm{~mm}$ $(-3 \mathrm{~dB})$ and $0.31 \mathrm{~mm}(-6 \mathrm{~dB})$ respectively, and the lateral resolution was $0.41 \mathrm{~mm}(-3 \mathrm{~dB})$ and $0.62 \mathrm{~mm}(-6 \mathrm{~dB})$. The experimental ultrasound scanning system RASMUS was used to verify the simulations. A wire phantom was used to measure the PSF. The wire was located $40 \mathrm{~mm}$ from the transducer surface. A BK8804 transducer was used with a center frequency of $7 \mathrm{MHz}$. The transmit and receiving aperture were identical to the simulated setups. The conventional STA technique can be seen in Fig. 8. The axial resolution was $0.25 \mathrm{~mm}(-3 \mathrm{~dB})$ and $0.38 \mathrm{~mm}(-6 \mathrm{~dB})$ respectively, and the lateral resolution was $0.71 \mathrm{~mm}(-3 \mathrm{~dB})$ and $1.06 \mathrm{~mm}(-6 \mathrm{~dB})$. The experiment was repeated using the frequency division approach with 16 bands starting from 1.5 MHz to $17.5 \mathrm{MHz}$. The PSF is given in Fig. 9. Axial resolution $0.21 \mathrm{~mm}(-3 \mathrm{~dB})$ and $0.29 \mathrm{~mm}(-$ $6 \mathrm{~dB})$ respectively, and lateral resolution $0.52 \mathrm{~mm}(-3 \mathrm{~dB})$ and $0.73 \mathrm{~mm}(-6 \mathrm{~dB})$.

\section{CONCLUSION}

A new method for STA imaging has been presented, where all elements can be excited at the same time and still be separated at the receiver. The method improves the $S N R$ by a factor of $N \frac{E_{s}}{E_{h}}$ and makes information from all elements available in every transmission and is therefore suitable for flow imaging. Both simulations and experiments show that the method is realizable in an ultrasound system.

\section{ACKNOWLEDGEMENT}

This work was supported by grant 9700883, 9700563 and 26-01-0178 from the Danish Science Foundation and by BK Medical A/S, Denmark.

\section{REFERENCES}

[1] S. I. Nikolov. Synthetic aperture tissue and flow ultrasound imaging. PhD thesis, Ørsted•DTU, Technical University of Denmark, 2800, Lyngby, Denmark, 2001.

[2] M. Karaman, P. C. Li, and M. O'Donnell. Synthetic aperture imaging for small scale systems. IEEE Trans. Ultrason., Ferroelec., Freq. Contr., 42:429-442, 1995.

[3] K. L. Gammelmark and J. A. Jensen. Multi-element synthetic transmit aperture imaging using temporal encoding. In Proc. SPIE: Progress in biomedical optics and imaging, volume 3, pages 25-36, 2002.

[4] R. Y. Chiao, L. J. Thomas, and S. D. Silverstein. Sparse array imaging with spatially-encoded transmits. In Proc. IEEE Ultrason. Symp., pages 1679-1682, 1997.

[5] S. Haykin. Communication Systems. John Wiley \& Sons, Inc., 2001.

[6] J. A. Jensen. Field: A program for simulating ultrasound systems. Med. Biol. Eng. Comp., 10th Nordic-Baltic Conference on Biomedical Imaging, Vol. 4, Supplement 1, Part 1:351-353, 1996b.

[7] J. A. Jensen, O. Holm, L. J. Jensen, H. Bendsen, H. M. Pedersen, K. Salomonsen, J. Hansen, and S. Nikolov. Experimental ultrasound system for real-time synthetic imaging. In Proc. IEEE Ultrason. Symp., volume 2, pages 1595-1599, 1999. 


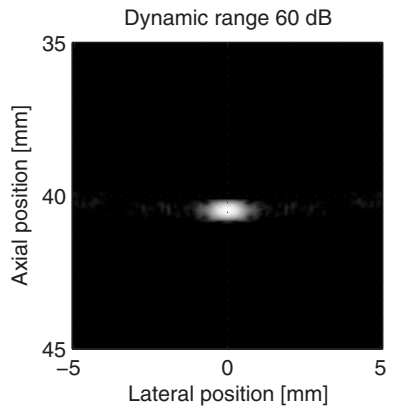

Figure 4: Simulated PSF for the STA system with 8 transmission centers

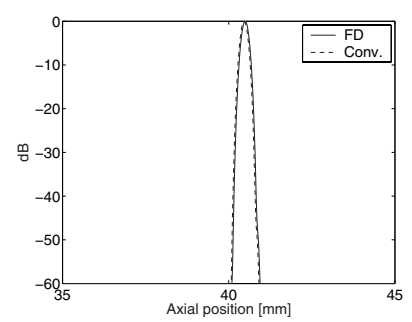

Figure 6: Axial projection of Fig. 4 and Fig. 5

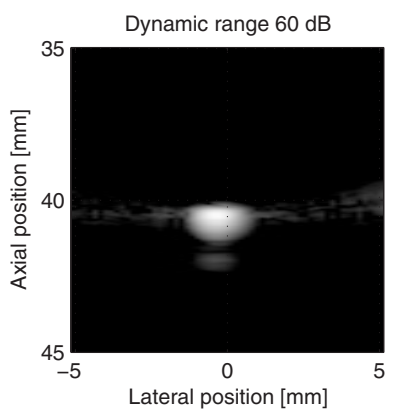

Figure 8: PSF for the STA system with 8 transmission centers

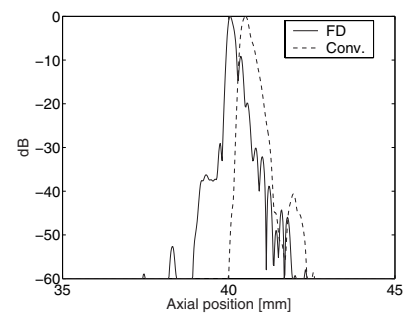

Figure 10: Axial projection of Fig. 8 and Fig. 9

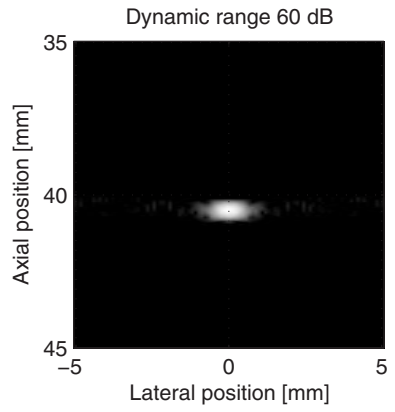

Figure 5: Simulated PSF for the FD system with 8 transmission centers

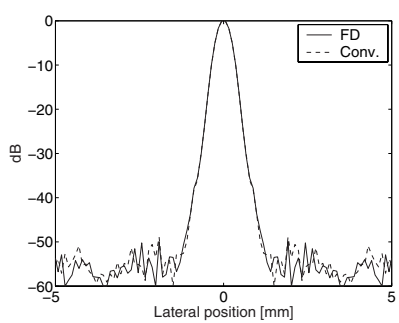

Figure 7: Lateral projection of Fig. 4 and Fig. 5

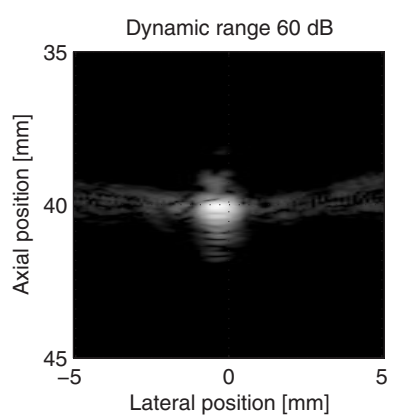

Figure 9: PSF for the FD system with 8 transmission centers

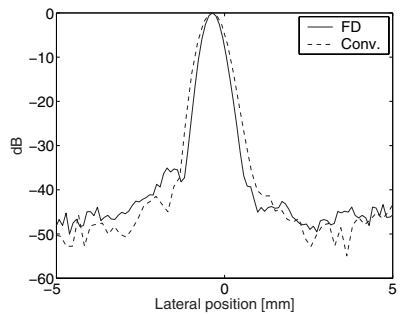

Figure 11: Lateral projection of Fig. 8 and Fig. 9 